\title{
Pre-operative hydrostatic reduction of intussusception in an adult
}

\author{
M I M De Zoysa ${ }^{1}$ and C Halahakoon ${ }^{2}$ \\ (Index words: intussusception, caecal tumour, hydrostatic reduction)
}

\section{Case report}

A 43-year old female had episodes of severe and worsening colicky pain confined to the right side of the abdomen for 3 days. The episodes of pain were not associated with vomiting or abdominal distension. She had no history of altered bowel habits, loss of appetite or progressive loss of weight. The pain was associated with a palpable sausage-shaped mass to the right of the umbilicus. Ultrasound scan of the abdomen was suggestive of an intussusception in the right iliac fossa. Contrast enhanced CT scan of the abdomen (Figure 1) confirmed the diagnosis of a right colo-colic intussusception. There were no liver metastases. During the last episode of colic, hydrostatic reduction of the intussusception was performed successfully. About two litres of normal saline was allowed to flow into the rectum through a rectal tube, keeping the container $1 \mathrm{~m}$ above the level of the patient with the foot end elevated. The patient was kept in that position until the pain subsided and the lump disappeared. Reduction was achieved in 35 minutes.

Colonoscopy performed 3 days later after bowel preparation revealed a polypoidal growth in the caecum. Histology showed a moderately differentiated adenocarcinoma. An elective right hemicolectomy was performed 10 days after reduction of intussusception. Histology revealed a $2 \mathrm{~cm}$ wide moderately differentiated adeno carcinoma which was involving the serosa (Dukes B, $\mathrm{pT}_{3} \mathrm{pN}_{0} \mathrm{pM}_{\mathrm{X}}$ ). She was given a course of 5-fluorouracil based chemotherapy. She was followed up with ultrasonography, colonoscopy and serum CEA and was free of local recurrence or metastatic disease six years after surgery.

\section{Discussion}

Intussusception is rare in adults with an incidence of around 2-3 per 100,000 per year. The use of investigations including a barium enema, ultrasound scan and computed tomography (CT) can be helpful to establish the diagnosis. The classical ultrasonic appearance is a doughnut sign in transverse view and pseudo-kidney sign on longitudinal view [1]. CT scan has a diagnostic accuracy of around $80 \%$. The classical finding on a CT scan is a target lesion which represents the outer intussuscepiens and the inner intussusceptum. The dense intussuscepted mass comprising of swollen bowel and mesentery within the lumen of the bowel is responsible for the characteristic target lesion seen on the CT scan [2]. Colonoscopy can be used in preoperative diagnosis although it is technically challenging [3].

In more than $90 \%$ of adults a lead point can be identified as a cause for the intussusception. This is usually a polyp or a tumour and in two thirds of these cases the colonic tumours are malignant. Old age and anaemia have been shown to be predictive of malignancy in multivariate analyses [4]. There was no reason to suspect a colonic malignancy in this patient as she was young and not anaemic.

In general, operative intervention is required in all cases of adult intussusception as conservative treatment is not effective [5]. However, in this patient we were able to reduce the intussusception successfully. As expected the volume of saline used was substantially higher than that required in children. It was possible to perform a semielective diagnostic colonoscopy after bowel preparation. Moreover, elective right hemicolectomy was planned after appropriate counselling. She was free of symptoms from the time of reduction to the time of surgery. A few authors have described intra-operative reduction of intussusception before resection as it helps in better planning of the extent of resection. However, most authors do not recommend this due to a higher incidence of malignancy in these cases and hence the risk of tumour embolisation and seeding [5]. This patient was disease free six years after surgery indicating that pre-operative reduction had been safe. ${ }^{1}$ Department of Surgery, Faculty of Medicine, University of Colombo, Sri Lanka and ${ }^{2}$ University Surgical Unit,
National Hospital of Sri Lanka.

Correspondence: MIMdeZ, e-mail <ishandz@hotmail.com>. Received 7 July and accepted 19 September 2009. Competing interests: none declared. 


\section{References}

1. Boyle MJ, Arkell LJ, Williams JT. Ultrasonic diagnosis of adult intussusception. American Journal of Gastroenterology 1993; 88: 617-8.

2. Gayer G, Zissin R, Apter S, et al. Pictorial review: adult intussusception - a CT diagnosis. British Journal of Radiology 2002, 75: 185-90.
3. Hurwitz LM, Gertler SL. Colonoscopic diagnosis of ileocaecal intussusception. Gastrointestinal Endoscopy 1986; 32: $217-8$

4. Goh BK, Quah HM, Chow PK, et al. Predictive factors of malignancy in adults with intussusception. World Journal of Surgery 2006; 30: 1300-4.

5. Azar T, Berger DL. Adult intussusception. Annals of Surgery 1997; 226: 134-8. 